\title{
BMJ Open Trend and risk factors of recurrence and complications after arrhythmias radiofrequency catheter ablation: a nation-wide observational study in Taiwan
}

Yuan Lin, ${ }^{1}$ Hsin-Kuan Wu, ${ }^{1}$ Te-Hsiung Wang, ${ }^{2}$ Tien-Hsing Chen, ${ }^{3,4}$ Yu-Sheng Lin ${ }^{5}$

To cite: Lin Y, Wu H-K, Wang $\mathrm{T}-\mathrm{H}$, et al. Trend and risk factors of recurrence and complications after arrhythmias radiofrequency catheter ablation: a nation-wide observational study in Taiwan. BMJ Open 2019;9:e023487. doi:10.1136/ bmjopen-2018-023487

- Prepublication history and additional material for this paper are available online. To view these files, please visit the journal online (http://dx.doi. org/10.1136/bmjopen-2018023487).

YL and H-KW contributed equally.

Received 23 April 2018 Revised 22 March 2019 Accepted 8 May 2019

Check for updates

(C) Author(s) (or their employer(s)) 2019. Re-use permitted under CC BY-NC. No commercial re-use. See rights and permissions. Published by BMJ.

For numbered affiliations see end of article.

\section{Correspondence to}

Dr Tien-Hsing Chen; skyheart0826@gmail.com and Dr Yu-Sheng Lin; dissertlin@gmail.com

\section{ABSTRACT}

Objectives This study determined the recurrence and complication rates after radiofrequency catheter ablation (RFCA) for those with paroxysmal supraventricular tachycardia (PSVT), Wolff-Parkinson-White syndrome (WPW), atrial flutter (AFL), atrial fibrillation (AF) and ventricular tachycardia (VT).

Study design and setting This retrospective study included RFCAs for 2001-2010 in the Taiwan National Health Insurance Research Database. Primary outcomes included perioperative complications (pericardial effusion and new-onset stroke), RFCA recurrence and long-term outcomes (high-grade atrioventricular block (AVB) and pacemaker implantation).

Results of 19,475 patients who underwent RFCA, prevalence rates were $56.7 \%$ for PSVT, $13.5 \%$ for WPW, 9.5\% for AFL, $5.1 \%$ for AF and $2.7 \%$ for VT. Prevalence rates increased in AF, AFL and VT over the study years. During an average follow-up period of 4.3 years (SD: 2.8 years), recurrence rates for PSVT, WPW, VT, AFL and AF were $2.0 \%, 4.9 \%, 5.7 \%, 5.8 \%$ and $16.1 \%$, respectively. Compared with the PSVT group, the WPW and AF groups had significantly higher risk of pericardial effusion during admission (adjusted OR (aOR) 2.98, 95\% Cl (Cl) 1.24 to 7.15; a0R $4.09,95 \% \mathrm{Cl} 1.90$ to 8.79 , respectively); the AFL group had a higher risk of new-onset stroke during admission (aOR 4.07, 95\% $\mathrm{Cl} 1.39$ to 11.91); the WPW group had a lower risk of high-grade AVB during follow-up (adjusted HR (aHR) $0.37,95 \% \mathrm{Cl} 0.19$ to 0.71 ) while the AFL group had a greater risk (aHR $1.74,95 \% \mathrm{Cl} 1.17$ to 2.60); and the AFL group had a higher risk of permanent pacemaker (aHR $2.14,95 \% \mathrm{Cl} 1.27$ to 3.62 ).

Conclusions The RFCA rate increased rapidly during 2001-2010 for AF, AFL and VT. Recurrence was associated with congenital heart disease in PSVT and WPW, and with age in AF and AFL. AFL had a higher risk of permanent pacemaker implantation and new stroke. AF had a higher risk of life-threatening pericardial effusion.

\section{INTRODUCTION}

Radiofrequency catheter ablation (RFCA) is used to treat patients with supraventricular tachycardia or ventricular tachycardia (VT),
Strengths and limitations of this study

- This 10-year longitudinal retrospective study is the first nation-wide, large-scale study of the trend, recurrence and complications of radiofrequency catheter ablation (RFCA).

- This article is the first study to compare recurrence and complications among five different types of arrhythmias after RFCA.

- Our study provides risks of arrhythmia recurrence and complications after RFCA.

- This study did not have access to certain data such as laboratory parameters, procedural details and heart images. Also, some arrhythmias such as premature ventricular beats and atrial premature beats are not covered by Taiwan National Health Insurance.

- This study was not able to explore the interactions among the predictive variables because of the limited number of events.

especially paroxysmal supraventricular tachycardia (PSVT). ${ }^{1-3}$ Widely applied since the 1990s, ${ }^{4}$ RFCA is an effective therapy with demonstrated high success, low complications and low recurrence rates compared with direct current ablation or surgical ablation. RFCA is superior to conservative treatments such as medication or observation for patients with PSVT and Wolff-Parkinson-White syndrome (WPW). RFCA was first used to treat atrial fibrillation (AF) in 1998.

Although arrhythmias after RFCA are usually not life-threatening, identification and minimisation of the risk of complications are extremely important. The RFCA procedure may lead to atrioventricular (AV) block and bradycardia, even requiring permanent pacemaker implantation. Previous studies ${ }^{5}$ were composed of relatively small cohorts or were single-centre studies and evaluated patients with a single arrhythmia. ${ }^{56}$ However, there are no studies comparing RFCA-related 
complications in patients with five different arrhythmias. ${ }^{78}$ The targets for RFCA-related risk minimisation differ by type of arrhythmia. For example, when RFCA is used to treat PSVT, the goal is to modify or eliminate $\mathrm{AV}$ node or accessory pathways; when used to treat $\mathrm{AF},{ }^{6}$ the goal is to isolate the pulmonary veins. High-grade AV block, life-threatening pericardial effusion and stroke are dangerous complications after an RFCA procedure. However, the complication rates vary by type of arrhythmia: PSVT, WPW, atrial flutter (AFL), AF and VT. It is therefore important to identify the incidence and risk factors of RFCA-related complications in these patients.

This retrospective study investigated the population trend of patients who received RFCA for PSVT, WPW, AFL, AF and VT. We identified the major RFCA-related risk factors influencing (1) recurrence of arrhythmias and (2) complications such as AV block, permanent pacemaker implantation, life-threatening pericardial effusion and acute ischaemic stroke.

\section{METHODS}

\section{Study design and population}

We conducted a nation-wide population-based cohort study using data from the Taiwan National Health Insurance Research Database (NHIRD). In Taiwan, the National Health Insurance (NHI) programme has reimbursed patients who receive RFCA for PSVT, WPW, AFL, AF and VT since 2001. More than 99.91\% of Taiwan's population is covered by NHI benefits. The accuracy and validation of NHIRD data are based on regular auditing by the NHI Bureau. ${ }^{9-11}$ The Institutional Review Board of Chang Gung Memorial Hospital approved this study.

\section{Study cohort, outcome measurement and follow-up}

This study accessed NHIRD data for all targeted patients with arrhythmia who received RFCA from 2001 to 2010. The targeted arrhythmias were PSVT (International Classification of Diseases, Ninth Revision, Clinical Modification (ICD-9 CM) Code 4270), WPW (426.7), AFL (427.32), AF (427.31) and VT (427.1; online supplementary table 1 ). Patients with arrhythmias other than those targeted (such as premature ventricular beats or atrial tachycardia) and patients with unidentified arrhythmias who received RFCA were excluded. We enrolled only the patient's first RFCA. The follow-up period was calculated from the discharge date of the index hospitalisation until death, loss to follow-up (withdrawal from the NHI programme: emigration or prison incarceration for longer than 6 months) or until the study end date (31 December 2010).

\section{Outcomes measurement}

The primary outcomes included recurrence of arrhythmia, in-hospital complications and long-term complications. Recurrence was defined as either (1) recurrence of original arrhythmia or (2) receipt of a second RFCA during the follow-up period. In-hospital complications included life-threatening pericardial effusion and new-onset stroke during the admission. Life-threatening pericardial effusion was defined as the patient requiring pericardiocentesis during RFCA. New stroke was defined as stroke (ICD-9 CM codes 430-437) which occurred during the index admission. Other complications included highgrade AV block and permanent pacemaker implantation.

\section{Covariate assessment}

Age was categorised into six groups $(0-18,19-44,45-54$, $55-64,65-74$ and 75 years and above) because previous studies reported different indications for RFCA and different complications between age groups. ${ }^{1-3}$ Comorbidities were assessed according to ICD-9 CM codes before the index admission. Diabetes mellitus, hypertension (HTN) or chronic diseases were recorded as comorbidities if there was at least one in-admission diagnosis. All congenital heart disease (CHD) was reconfirmed by the Catastrophic Illness Certification (CIC), which is a sub-dataset of NHI. A CIC for CHD requires imaging proof confirmed by two cardiologists. Complicated CHD included Tetralogy of Fallot (TOF), transposition of the great vessels, double outlet right ventricle, total anomalous pulmonary venous connection, tricuspid atresia, common truncus arteriosus, common ventricle and hypoplastic left heart syndrome. Simple CHD included ventricular septal defect (VSD), atrial septal defect, Ebstein's anomaly, patent ductus arteriosus, congenital pulmonary stenosis, coarctation of the aorta, endocardial cushion defect and congenital aortic stenosis. Centre activity was a time-dependent variable and a high-activity centre was defined as more than 100 RFCA surgeries per year, regardless of arrhythmia type.

\section{Patient and public involvement}

This study had no direct relationship with any patient and no public involvement during the development, design and conduct.

\section{Statistical analysis}

The proportion of categorical variables between groups was compared using the $\chi^{2}$ test and Fisher's exact test. Continuous variables were compared using Kruskal-Wallis test due to the lack of normality. Multivariable logistic regression analysis was used to identify clinical features associated with the risk of in-hospital complications, including life-threatening pericardial effusion and new-onset stroke during the admission. Multivariable Cox regression analysis was used to investigate the association of clinical variables with time-to-event outcomes, including recurrence, high-grade AV block and pacemaker implantation during the follow-up. In the survival analysis, the time-scale was time-since-RFCA in days. The assumption of proportional hazard was tested by Schoenfeld partial residuals, in which the indication was the only explanatory categorical variable. The 13 pre-specified potential predictive variables were those clinically relevant to RFCA and its complications: two demographic 
variables (sex and age), six comorbidities, four types of CHD and centre activity. All 13 candidate predictive variables were introduced into the multivariable regression models. Multicollinearity among predictors was checked by variance inflation factor (VIF), with a value less than 10 indicating no serious collinearity among predictors. Sensitivity analyses were done by excluding patients with recurrent RCFA during the follow-up (online supplementary table 2). A two-sided $\mathrm{p}$ value lower than 0.05 was considered statistically significant. No adjustment for multiple testing (multiplicity) was made in this study due to the limited size of event number. Results were presented as the OR for logistic regression, or HR for Cox regression with corresponding $95 \%$ CIs. All data analyses were performed using SPSS software V.15 (SPSS, Chicago, IL, USA).

\section{RESULTS}

There were 24003 RFCA procedures registered in NHIRD between 1 January 2001 and 31 December 2010. Based on the inclusion and exclusion criteria, a total of 19475 enrolled patients underwent 20707 RFCA procedures. Only the first occurrence for each individual was used for analysis. The proportion of change in rates of RFCA by individual arrhythmias from 2001 to 2010 is shown in figure 1. The proportion of RFCA for PSVT decreased from $60 \%$ to $51 \%$ between 2001 and 2010 , whereas the proportion for $\mathrm{AF}$ increased from $2 \%$ to $10 \%$ (figure 1 ).

The most common arrhythmia treated with RFCA was PSVT ( $\mathrm{n}=12796 ; 56.7 \%)$, followed by WPW $(\mathrm{n}=3051$; $13.5 \%)$, AFL $(\mathrm{n}=1854 ; 9.5 \%), \mathrm{AF}(\mathrm{n}=1162 ; 5.1 \%)$ and VT $(\mathrm{n}=612 ; 2.7 \%)$. The mean age of study participants when they received RFCA was 47.6 years (SD 18.3). Demographic and baseline clinical characteristics according to arrhythmia type are summarised in table 1 . The prevalence of PSVT (38.5\%), WPW $(58.1 \%)$ and VT (47.2\%) was highest in the group aged 19-44 years. Patients were the oldest in the AFL group, followed by the AF group, the PSVT group, the VT group and the WPW group. Patients with AF and AFL had a higher prevalence of diabetes $(16.2 \%$ and $11.5 \%$, respectively) and HTN (28.9\% and $28.1 \%$, respectively) compared with patients with other arrhythmias. Chronic obstructive pulmonary disease, chronic kidney disease (CKD) and heart failure were most prevalent in the AFL group since these patients were the oldest (median age 62.9 years). RFCA due to $\mathrm{AF}$ was predominantly performed in high-activity centres (84\%), followed by AFL (71\%). Complicated CHD was more common in the AFL group than in other arrhythmias. Simple CHD was most prevalent in the AFL group $(3.6 \%)$, followed by the VT group $(1.5 \%)$.

\section{Risk factors of recurrence}

During an average follow-up period of 4.3 years (SD 2.8 years), the recurrence rates after the index RFCA for those with PSVT, WPW, VT, AFL and AF were $2.0 \%$, $4.9 \%, 5.7 \%, 5.8 \%$ and $16.1 \%$, respectively. All VIF
The proportion of five arrhythmias in 2001

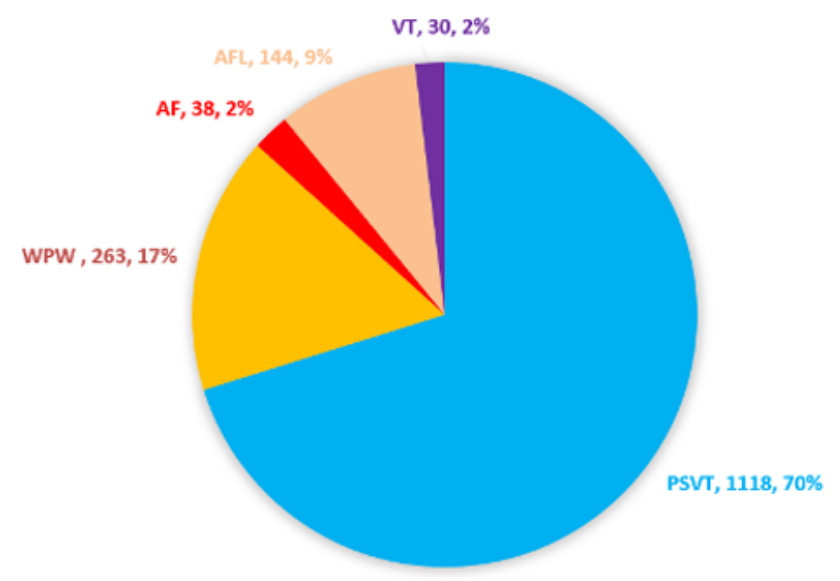

The proportion of five arrhythmias in 2010

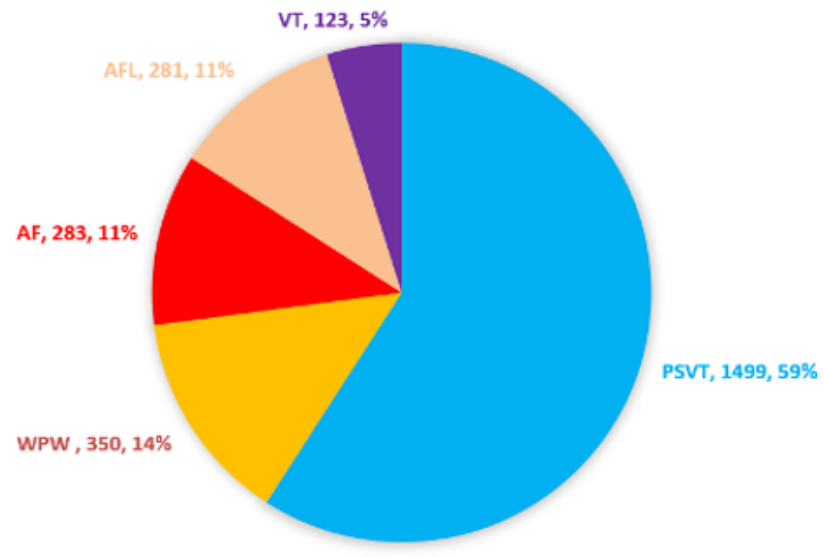

Figure 1 Proportion of radiofrequency catheter ablation patients diagnosed with PSVT, WPW, AFT, AF and VT in Taiwan during 2001 and 2010. AF, atrial fibrillation; AFT, atrial flutter; PSVT, paroxysmal supraventricular tachycardia; VT, ventricular tachycardia; WPW, Wolff-Parkinson-White syndrome.

values were less than 4 in the five multivariable models which indicated no apparent multicollinearity problem. Multivariable Cox analyses revealed that the major risk factors for recurrence of PSVT after RFCA included age (0-18 years), male gender, diabetes and TOF. Younger patients (0-18 vs 19-44 years) and those with Ebstein's anomaly were considered at greater risk for recurrence of WPW after RFCA (table 2). For the AFL group, older individuals (45-54 vs 19-44 years) had a higher risk of recurrence. Male gender, TOF, VSD and high-activity centre were also risk factors. In contrast, the incidence of AFL recurrence was low in patients 75 years or older. The recurrence rate was $16.1 \%$ in patients with $\mathrm{AF}$ but $2.0 \%$ for those with PSVT. The recurrence-free rate after RFCA declined with time, most sharply for those with AF (figure 2). Patients aged 19-44 years had a higher risk of $\mathrm{AF}$ recurrence compared with patients older than 65 years; male gender and treatment at a high-activity centre were also identified as risk factors. In the VT population, treatment at a high-activity centre was related to 
Table 1 Baseline data for 19475 study patients who underwent RFCA procedures stratified by indication

\begin{tabular}{|c|c|c|c|c|c|c|}
\hline Variable & PSVT & WPW & AFL & AF & VT & $P$ value \\
\hline Number of patients & 12796 & 3051 & 1854 & 1162 & 612 & - \\
\hline $\begin{array}{l}\text { Age (years), median } \\
\text { (IQR) }\end{array}$ & $47.0(33.5,58.6)$ & $36.3(22.8,49.7)$ & $62.9(51.7,73.1)$ & $56.9(48.4,65.5)$ & $43.1(28.7,55.2)$ & $<0.001$ \\
\hline Age group (years) & & & & & & $<0.001$ \\
\hline $0-18$ & $863(6.7)$ & $379(12.4)$ & $15(0.8)$ & $0(0.0)$ & $46(7.5)$ & \\
\hline $19-44$ & $4930(38.5)$ & $1619(53.1)$ & $260(14.0)$ & $216(18.6)$ & $289(47.2)$ & \\
\hline $55-64$ & $2083(16.3)$ & $308(10.1)$ & 407 (22.0) & $354(30.5)$ & 75 (12.3) & \\
\hline $65-74$ & $1344(10.5)$ & $130(4.3)$ & $472(25.5)$ & $222(19.1)$ & $51(8.3)$ & \\
\hline $75+$ & $638(5.0)$ & $36(1.2)$ & $371(20.0)$ & $85(7.3)$ & $28(4.6)$ & \\
\hline Gender, male & $5402(42.2)$ & $1988(65.2)$ & $1332(71.8)$ & $838(72.1)$ & $327(53.4)$ & $<0.001$ \\
\hline CKD & $150(1.2)$ & $12(0.4)$ & $71(3.8)$ & $11(0.9)$ & $5(0.8)$ & $<0.001$ \\
\hline CAD & $594(4.6)$ & 87 (2.9) & $288(15.5)$ & $154(13.3)$ & $45(7.4)$ & $<0.001$ \\
\hline Heart failure & $73(0.6)$ & $21(0.7)$ & $205(11.1)$ & $53(4.6)$ & $25(4.1)$ & $<0.001$ \\
\hline High-activity centre* & $7267(56.8)$ & $1880(61.6)$ & $1317(71.0)$ & $976(84.0)$ & $317(51.8)$ & $<0.001$ \\
\hline Complicated CHD & $10(0.1)$ & $3(0.1)$ & $16(0.9)$ & $2(0.2)$ & $1(0.2)$ & $<0.001$ \\
\hline TOF & $3(0.0)$ & $0(0.0)$ & $11(0.6)$ & $1(0.1)$ & $1(0.2)$ & $<0.001$ \\
\hline $\begin{array}{l}\text { Other complicated } \\
\text { CHD }\end{array}$ & $7(0.1)$ & $3(0.1)$ & $5(0.3)$ & $1(0.1)$ & $0(0.0)$ & 0.045 \\
\hline Simple CHD † & $69(0.5)$ & $31(1.0)$ & $66(3.6)$ & $9(0.8)$ & $9(1.5)$ & $<0.001$ \\
\hline VSD & $15(0.1)$ & $6(0.2)$ & $25(1.3)$ & $0(0.0)$ & $4(0.7)$ & $<0.001$ \\
\hline
\end{tabular}

*Defined as 100 operations per year.

†The discrepancy between the sums of subgroups and the total is due to the possibility that one patient might have two CHDs. AF, atrial fibrillation; AFL, atrial flutter; ASD, atrial septal defect; CAD, coronary artery disease; CHD, congenital heart disease; CKD, chronic kidney disease; COPD, chronic obstructive pulmonary disease; Ebstein, Ebstein's anomaly; PSVT, paroxysmal supraventricular tachycardia; RFCA, radiofrequency catheter ablation; TOF, Tetralogy of Fallot; VSD, ventricular septal defect; VT, ventricular tachycardia; WPW, Wolff-Parkinson-White syndrome.

decreased risk of recurrence. The results were similar when excluding patients with recurrent RCFA during the follow-up (online supplementary table 3 ).

\section{Complications}

Rates of RFCA-related complications were evaluated for the five arrhythmia groups (table 3). The overall rates of complications and mortality were less than $1 \%$ and $0.1 \%$, respectively. High-grade AV block was the most common complication following RFCA in all groups except the AF group. RFCA was more associated with life-threatening pericardial effusion in the AF group (1.3\%) than in the other groups. In the AFL group, RFCA was more associated with high-grade AV block (2.5\%), permanent pacemaker implantation $(1.4 \%)$ and new stroke $(0.5 \%)$ than in other groups.

\section{Risk factors for complications}

All VIF values were less than 4 in the four multivariable models which indicated no apparent multicollinearity problem. As to in-hospital complications, multivariable logistic regression revealed that age $>44$ years, high-activity centre and RFCA after WPW or AFL were associated with increased risk of life-threatening pericardial effusion. Age $>55$ years and RFCA after AFL were associated with a higher risk of stroke following RFCA (table 4). As to long-term complications, multivariable Cox regression identified the risk factors for high-grade AV block as age $\geq 75$ years, diabetes and heart failure. Patients with WPW were at a lower risk of developing AV block than patients with PSVT. Risk factors for pacemaker implantation were age $\geq 75$ years, diabetes, CKD and RFCA after AFL (when compared with PSVT). The 


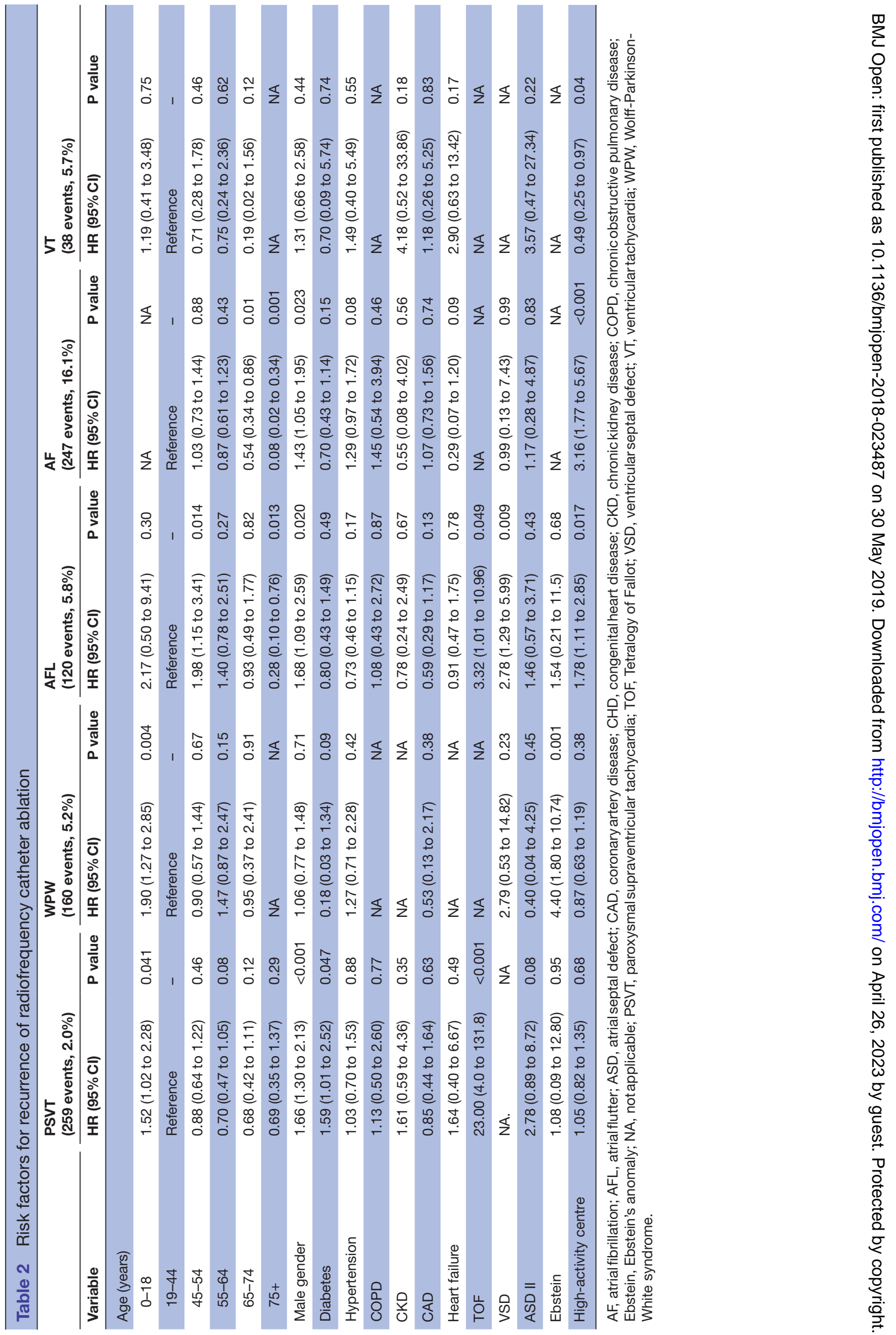


The recurrence-free survival curves for five arrhythmias

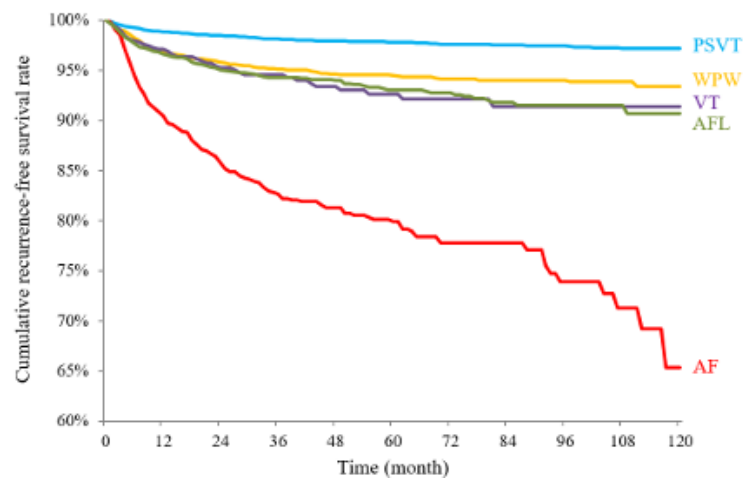

Figure 2 Recurrence-free survival curves after radiofrequency catheter ablation for groups of patients with initial diagnosis of paroxysmal supraventricular tachycardia, Wolff-Parkinson-White syndrome, atrial flutter, atrial fibrillation and ventricular tachycardia.

results were similar when excluding patients with recurrent RCFA during the follow-up (online supplementary table 4$)$.

Testing of Schoenfeld partial residuals revealed insignificant correlation for rank of survival time after AV block and permanent pacemaker implantation (AV block: number of events $=184, r=0.08, p=0.27$; permanent pacemaker implantation: number of events $=100, r=0.15$, $\mathrm{p}=0.11$ ), which indicated that the assumption of proportional hazard was not strongly violated (data not shown).

\section{DISCUSSION}

To the best of our knowledge, this is the first observational study to record the impact of RFCA on the treatment of arrhythmias by analysing the trends, risk factors, recurrence and complications of patients with five different arrhythmias. From 2001 to 2010, the number of RFCAs increased rapidly for the AF, AFL and VT groups, but decreased gradually for the PSVT and WPW groups. Age was a risk factor for recurrence in all groups, whereas male gender, diabetes and TOF were risk factors for recurrence in patients with PSVT. Patients with AF treated in a high-activity centre had a tendency to receive repeated RFCAs. Elderly patients with AF and AFL had more adverse events after RFCA compared with other subgroups.

\section{Trend in types of arrhythmias}

In Taiwan, the number of AF increased the most over the 10 years studied, followed by the VT, AFL, WPW and PSVT groups. Population ageing and advancements in ablation techniques have contributed to this phenomenon, especially for AF and AFL, which are age-related diseases. ${ }^{12}$ From 2001 to 2010, the population of older adult patients (>65 years) increased from 1973357 to 2487 893. This increase has resulted in a greater increase in the incidence of AF and AFL compared with other arrhythmias. The mean growth rate for RFCA per year between 2001 and 2010 was $9.7 \%$ for $\mathrm{AF}$ and $3.2 \%$ for AFL (figure 3). In contrast, the average growth rate of RFCA for PSVT was just $1.4 \%$, which was gradually slowing, although the absolute numbers increased from 1118 in 2001 to 1499 in 2010. This pattern is likely present for PSVT since (1) RFCA for PSVT is relatively mature compared with RFCA for AF, and (2) RFCA for PSVT was fully covered by Taiwan NHI but AF was not. Because patients with PSVT and WPW were relatively young, we searched the birth rate from 1980 to 2000. The crude birth rate (births per 1000 mid-year population per year; mid-year population is defined as the population on 30th June.) in Taiwan decreased from 413177 births (23 births per 1000 population) in 1980 to 307200 births (13 births per 1000 population) in 2000, reducing the number of patients needing PSVT and WPW. The number of WPW cases peaked in $2005(\mathrm{n}=377)$ and has since been decreasing. The number of procedures in the VT group increased from 57 in 2001 to 123 in 2010, and the average RFCA growth rate over 10 years was $6.8 \%$. This relatively high growth rate is possibly also due to population ageing and the maturation of three-dimensional mapping techniques. ${ }^{13}$ In summary, the growth models are different for the five arrhythmias. The AF and AFL groups have increased rapidly in RFCA procedures because of population ageing. The PSVT group had a relatively slow increase, whereas the WPW and VT groups showed stable or decreasing numbers of RFCAs.

\begin{tabular}{llllll}
\hline \multicolumn{7}{l}{ Table 3} & \multicolumn{7}{l}{ Numbers and rate of RFCA-related complications according to type of arrhythmias } \\
\hline Complication & PSVT & WPW & AFL & AF & VT \\
\hline $\begin{array}{l}\text { Number of patients } \\
\text { In-hospital complications (numbers and per cent) }\end{array}$ & 3051 & 1854 & 1162 & 612 \\
$\begin{array}{l}\text { Life-threatening pericardial effusion } \\
\text { New-onset stroke }\end{array}$ & $(15,0.12 \%)$ & $(8,0.26 \%)$ & $(6,0.32 \%)$ & $(15,1.30 \%)$ & $(1,0.16 \%)$ \\
$\begin{array}{l}\text { After discharge } \\
\text { High-grade AVB }\end{array}$ & $(1,0.06 \%)$ & $(2,0.07 \%)$ & $(9,0.49 \%)$ & $(4,0.34 \%)$ & $(0,0.00 \%)$ \\
Pacemaker implantation & $(64,0.50 \%)$ & $(5,0.16 \%)$ & $(26,1.40 \%)$ & $(2,0.17 \%)$ & $(3,0.50 \%)$
\end{tabular}

$\mathrm{AF}$, atrial fibrillation; AFL, atrial flutter; AVB, atrioventricularblock; PSVT, paroxysmal supraventricular tachycardia; RFCA, radiofrequency catheter ablation; VT, ventricular tachycardia; WPW, Wolff-Parkinson-White syndrome. 


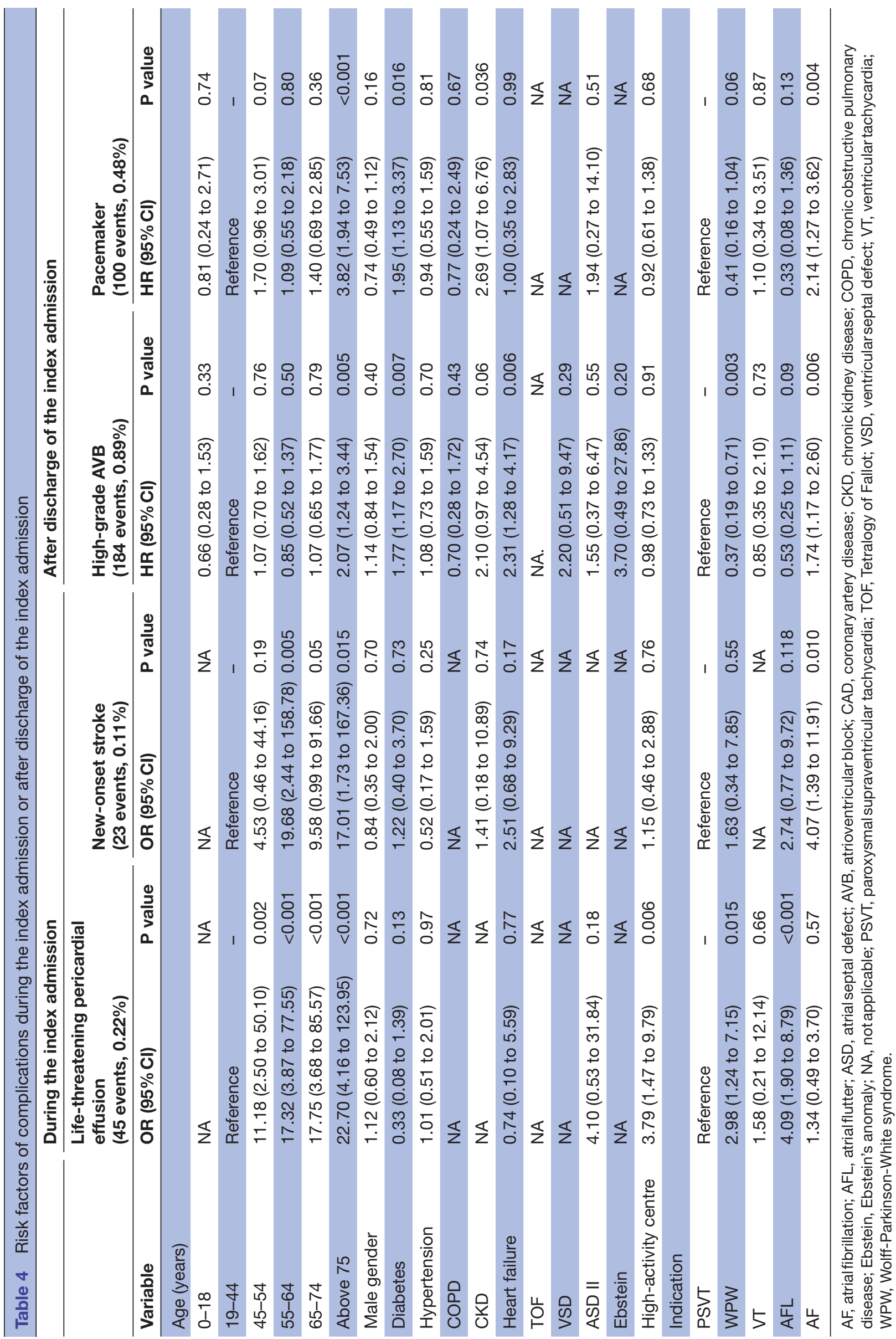


Annual RCFA numbers for PSVT, WPW, AFL, AF, and VT

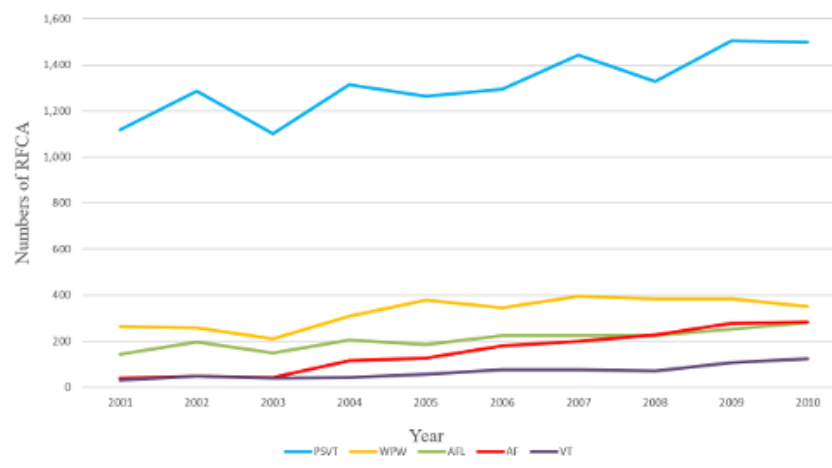

Annual RCFA growth rate for PSVT, WPW, AFL, AF, and VT

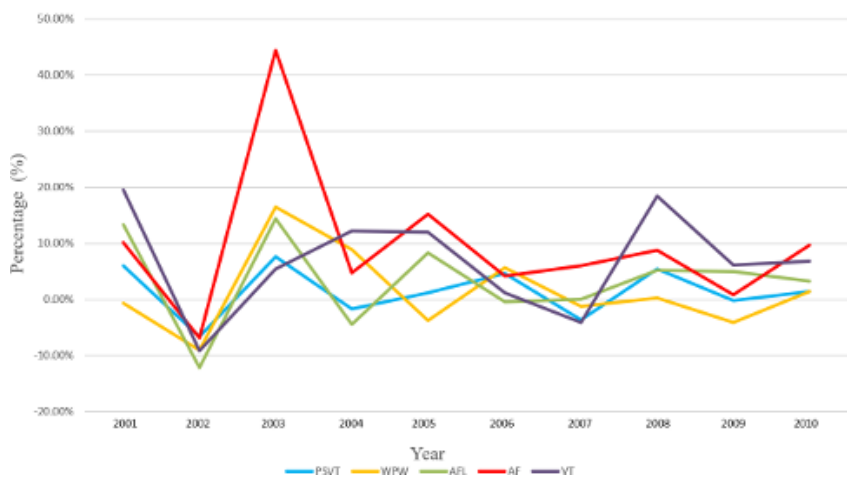

Figure 3 Numbers and growth rate of radiofrequency catheter ablations annually in groups of patients with initial diagnosis of PSVT, WPW, AFL, AF and VT. AF, atrial fibrillation; AFL, atrial flutter; PSVT, paroxysmal supraventricular tachycardia; RCFA, $r$ adiofrequency catheter ablation; VT, ventricular tachycardia; WPW, Wolff-Parkinson-White syndrome.

\section{Risk of recurrence}

Our results showed that the recurrence rate after RFCA increased in the following order: PSVT $(2.0 \%)<\mathrm{WPW}$ $(4.9 \%)<\operatorname{VT}(5.7 \%)<\operatorname{AFL}(5.8 \%)<\operatorname{AF}(16.1 \%)$ (figure 2$)$. The recurrence-free rate was highest for the PSVT group (98.8\% for the first year, gradually decreasing to $97.2 \%$ for the 10-year follow-up). However, patients $<18$ years in the PSVT and WPW groups had a significantly higher chance of recurrence, a result which agreed with those of Van Hare et $a l^{14}$ This recurrence could be a result of the smaller cardiac anatomy in children, which makes the precise ablation difficult to perform. This result could also explain the association of CHD and TOF with recurrence of PSVT, possibly because of the abnormal cardiac structure of the CHD heart postcardiac surgery. Patients with $\mathrm{TOF}$ and $\mathrm{AF}$ also had a higher risk of receiving a second RFCA. In contrast, AF and AFL patients aged $\geq 75$ years had fewer second RFCAs than younger patients. ${ }^{15}$

Our data showed that patients $>75$ years receiving treatment for AF and AFL had lower recurrence rates than those the same age in other groups. The reason for this phenomenon may be the conservative treatment preferred by cardiologists for older patients rather than repeated RFCA, in order to avoid complications or mortality after the first procedure due to the presence of other comorbidities. Our data suggest that, for patients undergoing an elective RFCA, physicians should carefully evaluate the risk factors such as younger age and the presence of CHD (TOF in PSVT, VSD in AFL) which are associated with a high recurrence rate. Our study also described epidemiological changes in repeated ablation procedures for five arrhythmias in Taiwan in the RFCA era.

\section{Complications}

RFCA, which has an approximately $1 \%$ complication rate and $0.1 \%$ mortality rate, ${ }^{316}$ is considered a relatively safe procedure to treat or even cure arrhythmias (table 3). Our present study showed different patterns of complications in the five arrhythmia groups. Patients with PSVT and WPW had complication rates of $1.6 \%$ and $0.8 \%$, respectively, similar to previous studies. However, in patients with $\mathrm{AF}$ and AFL, the complication rates were $2.5 \%$ and $4.7 \%$, respectively. AFL after RFCA induced more high-grade AV block $(2.5 \%)$ compared with other arrhythmias, and patients with AF after RFCA had the highest incidence rate of life-threatening pericardial effusion (1.3\%). Highgrade AV block is considered the main complication of ablation procedures for patients with AFL and PSVT because the ablation sites are close to the AV node. ${ }^{16}$ AFL has been seen combined with sick sinus syndrome. Bradyarrhythmias appeared when the substance of AF and AFL is eliminated. Patient who underwent RFCA for AF had a higher risk of life-threatening pericardial effusion relative to patients with other arrhythmias, resulting in a relatively higher complication rate of $1.3 \%$. The major RFCA procedure for $\mathrm{AF}$ is to isolate the pulmonary vein and eliminate the substrate in the left atrium. This requires a longer procedure time and delivers more energy to convert AF into sinus rhythm. RFCA for AF could therefore cause more life-threatening pericardial effusion than that for other arrhythmias. RFCA for VT presents same pattern as that for PSVT and WPW. These data suggest that, although RFCA is a common procedure to treat different arrhythmias, the complications that should be monitored will differ by type of arrhythmia.

Our data also showed that patients with AFL and AF had higher stroke rates $(0.49 \%$ and $0.34 \%$, respectively) than patients in the other groups. Anticoagulation therapy is needed in these cases, and it is also necessary to confirm the absence of intracardiac thrombus before RFCA. ${ }^{17}$ However, anticoagulation procedures are sometimes ignored because anticoagulation is not routinely used in AFL. ${ }^{18}$ Previous studies have shown a high risk of thromboembolic events and a high incidence of thrombogenic milieu in AFL. ${ }^{1920}$ Use of the inappropriate anticoagulation therapy is considered a significant risk factor for thromboembolism in patients with AFL. ${ }^{18}$

Age was an important risk factor associated with complications such as high-grade AV block, pacemaker implantation, life-threatening pericardial effusion and stroke, especially in patients aged $\geq 75$ years (table 4 ). These data were consistent with previous studies, ${ }^{21} 22$ and suggest that physicians should be cautious when performing RFCA in 
patients $>75$ years. We also found that diabetes was associated with increased complication rates for RFCA. A cohort study of 200000 patients with type II diabetes reported that third-degree AV block was 3.1 times as prevalent in the group with diabetes (95\% CI, 3.0 to $3.3 ; \mathrm{p}<0.0001) .{ }^{23}$ Diabetes has been suggested as a risk factor for autonomic neuropathy, cardiac conduction abnormalities and bradyarrhythmias. ${ }^{24}$ Physicians performing RFCA in patients with diabetes should monitor for bradycardia complications.

\section{Limitations}

First, the major limitation of this study is our inability to explore the interactions among the predictive variables because of the limited number of events. For instance, the 184 high-grade AV blocks allow for a maximum of 18-19 predictive variables, due to the 'ten-one rule'. ${ }^{25-27}$ However, the 13 predictors indicate that 78 two-way potential interaction effects may exist. Therefore, it seems not feasible to perform a regression analysis (logistic or Cox regressions) because that many explanatory variables in the equation would induce the statistical problem of overfitting. In addition, there is also an issue of multiple testing (five tests in table 2 and four tests in table 4) in this study. Many of the results would turn to be statically insignificant if a correction (ie, Bonferroni adjustment) was done. Therefore, further studies with a larger sample size and more events are needed to conduct interaction tests based on clinical knowledge or on exploratory data analysis along with multiple testing correction.

Second, in this cohort study, we did not have access to laboratory parameters, procedural details, heart images, smoking status, obesity or alcohol use. Procedure-related parameters, the location of the accessory pathway in WPW, PV isolation for AF, cardiac anomaly and ejection fraction have been reported as predictors for arrhythmia recurrence and RFCA complications. ${ }^{1428-30}$ The lack of this information could induce residual confounding. On the other hand, the different arrhythmia groups had substantial differences in baseline characteristics, especially in terms of age, which may result in potential confounding even if we adjusted for these variables in the multivariable regression models. However, the present study focused on RFCA for five different arrhythmias and each arrhythmia had different surgical parameters. Rather than comparing the same parameter in different arrhythmia ablation procedures, we focused on the effect of comorbidities, gender and age on arrhythmia recurrence and RFCA-related complications. Our study provided valuable information to help cardiologists deal with RFCA recurrence and complications.

Third, some arrhythmias such as premature ventricular beats and atrial premature beats are not covered by Taiwan NHI. However, excluding these arrhythmias did not influence the study results since they are usually benign.

Lastly, recurrence may be misidentified in this present study as resulting from ablation of other arrhythmias. For example, this could happen if the patient had an initial PSVT ablation followed by an AF ablation. A single definition of recurrence could consider the second ablation as the recurrence of PSVT. Use of double criteria, with repeated ablations combined with the same major principal diagnosis, reduced the coding error in this study.

\section{CONCLUSIONS}

There was a rapidly increasing trend of RFCA procedures for AF, AFL and VT during 2001-2010, but a slow increase for PSVT and WPW. The recurrence-free rate was higher for PSVT than for other arrhythmias. Older adult patients with AF and AFL had fewer repeat RFCAs and patients with AF in high-activity centre hospitals had more. CHD was a risk factor for PSVT recurrence. Patients with AF had more occurrences of life-threatening pericardial effusion, especially those aged more than 65 years, and patients receiving RFCA for AFL suffered more from bradycardia, requiring permanent pacemaker implantation.

\section{Author affiliations}

${ }^{1}$ Emergency Medicine Department, Chang Gung Memorial Hospital Keelung Branch, Keelung, Taiwan

${ }^{2}$ Department of Primary Care and Emergency Medicine, Kyoto University Graduate School of Medicine Faculty of Medicine, Kyoto, Japan

${ }^{3}$ Division of Cardiology, Department of Internal Medicine, Chang Gung Memorial Hospital Keelung Branch, Keelung, Taiwan

${ }^{4}$ Biostatistical Consultation Center, Chang Gung Memorial Hospital Keelung Branch, Keelung, Taiwan

${ }^{5}$ Division of Cardiology, Department of Internal Medicine, Chiayi Chang Gung Memorial Hospital, Puzi, Chiayi, Taiwan

Collaborators CHEN conceived of the study. Y LIN and WU initiated the study design and WANG helped with implementation. Y LIN, WU and CHEN provided statistical expertise in clinical trial design and WANG and YS LIN conducted the primary statistical analysis. All authors contributed to refinement of the study protocol and approved the final manuscript.

Funding This work was supported by grants from the Chang Gung Memorial Hospital, Taiwan (CGRPG2F0011, CLRPG2C0021, CLRPG2C0022, CLRPG2C0023, CLRPG2C0024, CLRPG2G0081, CLRPG2G0082 and CLRPG2H0041).

Disclaimer The lead author confirms that the content of this manuscript is honest and transparent.

Competing interests None declared.

Patient consent for publication Not required.

Ethics approval The Ethics Institutional Review Board at Chang Gung Memorial Hospital approved this study.

Provenance and peer review Not commissioned; externally peer reviewed.

Data sharing statement Data are available. Please contact the corresponding authors.

Open access This is an open access article distributed in accordance with the Creative Commons Attribution Non Commercial (CC BY-NC 4.0) license, which permits others to distribute, remix, adapt, build upon this work non-commercially, and license their derivative works on different terms, provided the original work is properly cited, appropriate credit is given, any changes made indicated, and the use is non-commercial. See: http://creativecommons.org/licenses/by-nc/4.0/.

\section{REFERENCES}

1. O'Hara GE, Philippon F, Champagne J, et al. Catheter ablation for cardiac arrhythmias: a 14-year experience with 5330 consecutive patients at the Quebec Heart Institute, Laval Hospital. Can J Cardiol 2007;23 Suppl B:140. 
2. Spector P, Reynolds MR, Calkins H, et al. Meta-analysis of ablation of atrial flutter and supraventricular tachycardia. Am J Cardiol 2009;104:671-7.

3. Bohnen M, Stevenson WG, Tedrow UB, et al. Incidence and predictors of major complications from contemporary catheter ablation to treat cardiac arrhythmias. Heart Rhythm 2011;8:1661-6.

4. Joseph JP, Rajappan K. Radiofrequency ablation of cardiac arrhythmias: past, present and future. QJM 2012;105:303-14.

5. Cosío FG. Atrial flutter, typical and atypical: A review. Arrhythm Electrophysiol Rev 2017;6:55-62.

6. Nyong J, Amit G, Adler AJ, et al. Efficacy and safety of ablation for people with non-paroxysmal atrial fibrillation. Cochrane Database Syst Rev 2016;11:CD012088.

7. Pérez FJ, Schubert CM, Parvez B, et al. Long-term outcomes after catheter ablation of cavo-tricuspid isthmus dependent atrial flutter: a meta-analysis. Circ Arrhythm Electrophysiol 2009;2:393-401.

8. Cappato R, Calkins H, Chen SA, et al. Updated worldwide survey on the methods, efficacy, and safety of catheter ablation for human atrial fibrillation. Circ Arrhythm Electrophysiol 2010;3:32-8.

9. Yang YW, Chen YH, Xirasagar S, et al. Increased risk of stroke in patients with bullous pemphigoid: a population-based follow-up study. Stroke 2011;42:319-23.

10. Wu CY, Wu MS, Kuo KN, et al. Effective reduction of gastric cancer risk with regular use of nonsteroidal anti-inflammatory drugs in Helicobacter pylori-infected patients. J Clin Oncol 2010;28:2952-7.

11. Wu CY, Chen YJ, Ho HJ, et al. Association between nucleoside analogues and risk of hepatitis $B$ virus-related hepatocellular carcinoma recurrence following liver resection. JAMA 2012;308:1906-14.

12. Feinberg WM, Blackshear JL, Laupacis A, et al. Prevalence, age distribution, and gender of patients with atrial fibrillation. Analysis and implications. Arch Intern Med 1995;155:469-73.

13. Dixit S, Callans DJ. Mapping for ventricular tachycardia. Card Electrophysiol Rev 2002;6:436-41.

14. Van Hare GF, Javitz H, Carmelli D, et al. Prospective assessment after pediatric cardiac ablation: recurrence at 1 year after initially successful ablation of supraventricular tachycardia. Heart Rhythm 2004;1:188-96.

15. Tuan TC, Chang SL, Tsao HM, et al. The impact of age on the electroanatomical characteristics and outcome of catheter ablation in patients with atrial fibrillation. J Cardiovasc Electrophysiol 2010;21:966-72.

16. Walters TE, Kistler PM, Kalman JM. Radiofrequency ablation for atrial tachycardia and atrial flutter. Heart Lung Circ 2012;21:386-94.

17. Calkins $\mathrm{H}$, Kuck KH, Cappato R, et al. HRS/EHRA/ECAS exper consensus statement on catheter and surgical ablation of atrial fibrillation: recommendations for patient selection, procedural techniques, patient management and follow-up, definitions, endpoints, and research trial design. J Interv Card Electrophysiol 2012;2012:171-257.

18. Grönefeld GC, Wegener F, Israel CW, et al. Thromboembolic risk of patients referred for radiofrequency catheter ablation of typical atrial flutter without prior appropriate anticoagulation therapy. Pacing Clin Electrophysiol 2003;26:323-7.

19. Wood KA, Eisenberg SJ, Kalman JM, et al. Risk of thromboembolism in chronic atrial flutter. Am J Cardiol 1997;79:1043-7.

20. Alyeshmerni D, Pirmohamed A, Barac A, et al. Transesophageal echocardiographic screening before atrial flutter ablation: is it necessary for patient safety? J Am Soc Echocardiogr 2013;26:1099-105

21. Hoffmann BA, Brachmann J, Andresen D, et al. Ablation of atrioventricular nodal reentrant tachycardia in the elderly: results from the German Ablation Registry. Heart Rhythm 2011;8:981-7.

22. Mirza M, Strunets A, Shen WK, et al. Mechanisms of arrhythmias and conduction disorders in older adults. Clin Geriatr Med 2012;28:555-73.

23. Movahed MR, Hashemzadeh M, Jamal MM. Increased prevalence of third-degree atrioventricular block in patients with type II diabetes mellitus. Chest 2005;128:2611-4.

24. Movahed MR. Diabetes as a risk factor for cardiac conduction defects: a review. Diabetes Obes Metab 2007;9:276-81.

25. Peduzzi P, Concato J, Kemper E, et al. A simulation study of the number of events per variable in logistic regression analysis. J Clin Epidemiol 1996;49:1373-9.

26. Concato J, Peduzzi P, Holford TR, et al. Importance of events per independent variable in proportional hazards analysis. I. Background, goals, and general strategy. J Clin Epidemiol 1995;48:1495-501.

27. Peduzzi P, Concato J, Feinstein AR, et al. Importance of events per independent variable in proportional hazards regression analysis. II. Accuracy and precision of regression estimates. J Clin Epidemiol 1995;48:1503-10.

28. Adao L, Araujo C, Ap S, et al. Importancia da posicao anatomica da via acessoria na eficacia e na seguranca da ablacao por radiofrequencia. Rev Port Cardiol 2011;30:35-46.

29. Iturralde P, Guevara-Valdivia M, Rodríguez-Chávez L, et al. Radiofrequency ablation of multiple accessory pathways. Europace 2002:4:273-80.

30. Anselmino M, Grossi S, Scaglione M, et al. Long-term results of transcatheter atrial fibrillation ablation in patients with impaired left ventricular systolic function. J Cardiovasc Electrophysiol 2013;24:24-32. 